BULL. AUSTRAL. MATH. SOC.

VOL. $19(1978),|6|-|8|$.

\title{
On the absolute Nörlund summability factors of a Fourier series
} and its conjugate series at a point

\section{Kösi Kanno}

The object of this paper is to give generalizations of Okuyama's Theorem [Bull. Austral. Math. Soc. 12 (1975), 9-2l, Tônoku Math. J. (2) 28 (1976), 563-581] on the absolute Nörlund summability factors of a Fourier series and its conjugate series.

Our theorems imply many results proved by other authors:

especially Theorem $I$ includes the results of Bhatt and Kishor [Indian J. Math. 9 (1967), 259-267 (1968)], Dikshit [Pacific J. Math. 63 (1976), 371-379], and Lal [Publ. Inst. Math. (Beorrad) 20 (34) (1976), 169-178], and we can easily deduce Lal's result [Indian J. Math. 16 (1974), 1-22] from our Corollary 2.

\section{Notations and theorems}

Let $\sum a_{n}$ be a given infinite series with the sequence of partial sums $\left\{s_{n}\right\}$. Let $\left\{p_{n}\right\}$ be a given sequence of constants, real or complex, such that $P_{n}=\sum_{k=0}^{n} p_{k} \neq 0$ for $n \geq 0$ and $p_{n}=P_{n}=0$ for $n<0$.

The sequence $\left\{t_{n}\right\}$ given by Izumi.

Received 10 July 1978. Communicated by Drs Shin-ichi and Masako 


$$
t_{n}=\frac{1}{P_{n}} \sum_{k=0}^{n} p_{n-k} s_{k}=\frac{1}{P_{n}} \sum_{k=0}^{n} P_{k} a_{n-k}
$$

defines the Nörlund means of the sequence $\left\{s_{n}\right\}$ generated by the sequence $\left\{p_{n}\right\}$. The series $\sum a_{n}$ is said to be absolutely summable $\left(N, p_{n}\right\}$, or summable $\left|N, p_{n}\right|$, if the sequence $\left\{t_{n}\right\}$ is of bounded variation, that is, if

$$
\sum_{n=1}^{\infty}\left|t_{n}-t_{n-1}\right|
$$

is convergent.

Let $f(t)$ be a periodic function with period $2 \pi$ and integrable over $(-\pi, \pi)$. Without any loss of generality we may assume that the constant term in the Fourier series of $f(t)$ is zero, so that

$$
f(t) \sim \sum_{n=1}^{\infty}\left(a_{n} \cos n t+b_{n} \sin n t\right) \equiv \sum_{n=I}^{\infty} A_{n}(t)
$$

and

$$
\int_{-\pi}^{\pi} f(t) d t=0
$$

The conjugate series to series (1.3) is

$$
\sum_{n=1}^{\infty}\left(b_{n} \cos n t-a_{n} \sin n t\right)=\sum_{n=1}^{\infty} B_{n}(t) .
$$

In what follows, we use the following notations:

$$
\begin{aligned}
& \varphi_{x}(t)=\varphi(t)=\frac{3}{2}\{f(x+t)+f(x-t)\} ; \\
& \Phi_{\alpha}(t)=\frac{1}{\Gamma(\alpha)} \int_{0}^{t}(t-u)^{\alpha-1} \varphi(u) d u \quad(\alpha>0) ; \\
& \Phi_{0}(t)=\varphi(t) ; \\
& h(u)=\int_{u}^{\pi}(t-u)^{-\alpha} \cos k t d t ;
\end{aligned}
$$




$$
\begin{aligned}
P(n, k) & =\frac{P_{n-k}}{P_{n}}-\frac{P_{n-k-1}}{P_{n-1}} ; \\
G_{\beta-\alpha}(n ; j, \tau, t) & =\sum_{k=j}^{I} P(n, k) \lambda_{k} \mu_{k} k^{\beta-\alpha} \cos k t ; \\
H_{B-\alpha}(n ; j, \tau, u) & =\frac{2}{\pi \Gamma(1-\alpha)} \int_{u}^{\pi}(t-u)^{-\alpha} G_{\beta-\alpha}(n ; j, \tau, t) d t ; \\
I(n ; j, \tau, u) & =\int_{0}^{u} v^{\beta} \frac{d}{d v} H_{\beta-\alpha}(n ; j, \tau, v) d v .
\end{aligned}
$$

Moreover we write

$$
\begin{aligned}
\psi_{x}(t) & =\psi(t)=\frac{z}{2}\{f(x+t)-f(x-t)\} ; \\
\tilde{G}_{B-\alpha}(n ; j, \tau, t) & =\sum_{k=j}^{l} P(n, k) \lambda_{k} \mu_{k} k^{\beta-\alpha} \sin k t .
\end{aligned}
$$

We employ $\Psi_{\alpha}(t), \tilde{H}_{\beta-\alpha}(n ; j, \imath, u)$ with meanings similar to the above notations. Throughout the present paper we denote by $\mu(t)$ a positive bounded function, $\lambda(t)$ a positive non-decreasing function, and $\left\{p_{n}\right\}$ a non-negative, non-increasing sequence.

Given a function $\omega(t)$, we write for $n=1,2, \ldots$,

$$
\omega(n)=\omega_{n}, \quad \Delta \omega_{n}=\omega_{n}-\omega_{n+1} .
$$

Let $[x]$ denote the greatest integer not greater than $x$; in particular we write $m=[n / 2]$ and $\tau=\left[\frac{2}{2}((2 \pi / u)-1)\right]$; and $A$ denotes a positive constant which is not necessarily the same at each occurrence.

The purpose of this paper is to establish some generalizations of Okuyama's results $[10],[11]$.

THEOREM 1. Let the sequence $\left\{\lambda_{n^{\mu} n^{\prime}} /(n+1)^{1-\beta}\right\}(0 \leq \beta \leq 1)$ be nonincreasing.

If the conditions

$$
\sum_{k=n}^{\infty} \frac{\lambda_{k} \mu_{k}}{k^{1-\beta_{P_{k}}}}=0\left(\frac{\lambda_{n^{n^{\beta}}}}{P_{n}}\right) \quad(n=1,2, \ldots),
$$




$$
\sum_{k=1}^{\infty} \frac{\lambda_{k} \mu_{k}}{k}\left|\cos \frac{\pi}{2}(\beta-\alpha+1)\right|<\infty,
$$

and

$$
\int_{0}^{\pi} \lambda(2 \pi / t)\left|d\left\{t^{-\beta_{\Phi}}(t)\right\}\right|<\infty
$$

hold for $0 \leq \alpha \leq \beta \leq 1$, then the series

$$
\sum_{n=1}^{\infty} \lambda_{n}{ }^{\mu} n^{n^{\beta-\alpha}} A_{n}(t)
$$

is sumable $\left|N, p_{n}\right|$ at $t=x$.

If $P_{n} \leq A \lambda_{n} n^{B}$, the right-hand side of condition (1.4) is replaced by $O(1)$.

This theorem has wider applications than the results of Bhatt and Kishore [1], Dikshit [2], and Lal [5], [6]. As special cases of Theorem 1 we obtain the results of Matsumoto [7].

THEOREM 2. Let $\left\{\Delta p_{n}\right\}$ be non-negative non-increasing. Assume that $n \lambda_{n} \mu_{n}, n^{I-\beta} / \lambda_{n} \mu_{n}$, and $\lambda_{n} \mu_{n} / p_{n}$ are all non-decreasing, where $0 \leq \beta \leq 1$. If the conditions (1.4),

$$
\int_{0}^{\pi} \frac{\lambda(2 \pi / t) \mu(2 \pi / t)}{t^{1+\beta}}\left|\Psi_{\alpha}(t)\right| d t<\infty,
$$

and

$$
\int_{0}^{\pi} \frac{\lambda(2 \pi / t)}{t^{\beta}}\left|d \Psi_{\alpha}(t)\right|<\infty
$$

hold for $0 \leq \alpha \leq \beta \leq 1$, then the series

$$
\sum_{n=1}^{\infty} \lambda_{n} \mu_{n} n^{\beta-\alpha_{B}}(t)
$$

is summable $\left|N, p_{n}\right|$ at $t=x$.

THEOREM 3. Let $\left\{\lambda_{n} \mu_{n} / n^{1-\beta}\right\}$ be a non-increasing sequence. If the 
conditions (1.4),

$$
\int_{0}^{\pi} \frac{\lambda(2 \pi / t)}{t^{\beta}}\left|d \Psi_{\alpha}(t)\right|<\infty \text {, and } \Psi_{\alpha}(+0)=0
$$

hold for $0 \leq \alpha \leq \beta \leq 1$, then the series

$$
\sum_{n=1}^{\infty} \lambda_{n} \mu_{n} n^{\beta-\alpha_{B}}(t)
$$

is summable $\left|N, p_{n}\right|$ at $t=x$.

In the two theorems above, if $P_{n} \leq A \lambda_{n} n^{B}$, then the right-hand side of $(1.4)$ is replaced by $O(1)$.

If the property

$$
\frac{d}{d t} \lambda(2 \pi / t)=A \frac{\lambda(2 \pi / t) \mu(2 \pi / t)}{t}
$$

holds for a suitable constant $A$, it is easy to see that (1.9) implies $(1.7)$.

\section{Proof and corollaries of Theorem 1}

We need some lemmas for the proof of Theorem 1 .

LEMMA 1. Let $\left\{a_{n}\right\}$ be a given sequence; then for any $x$, we have

$$
\text { (1-x) } \sum_{k=r}^{s} a_{k} x^{k}=a_{p} x^{r}-a_{s} x^{s+1}-\sum_{k=r}^{s-1} \Delta a_{k} x^{k+1} \text {, }
$$

where $r$ and $s$ are integers such tinat $s \geq r \geq 0$.

LEMMA 2. For $0 \leq a \leq b \leq \infty$ and any $n$,

$$
\left|\sum_{k=a}^{b} p_{k} \exp i(n-k) u\right| \leq A P_{\tau}
$$

uniformly in $0 \leq u \leq \pi$.

LEMiNA 3. For all $k \geq 0$ and $1 \leq a \leq b \leq \infty$,

$$
\sum_{n=a}^{b} P(n, k)=\sum_{n=a}^{b}\left(\frac{P_{n-k}}{P_{n}}-\frac{P_{n-k-1}}{P_{n-1}}\right) \leq 1 .
$$


The proofs of the above lemmas are quite easy and we omit them.

LEMMA 4. For $0<t \leq \pi$, and $0 \leq \alpha \leq \beta \leq 1$,

$$
\int_{0}^{t} u^{\beta-\alpha} \cos k u d u=\frac{t^{\beta-\alpha} \operatorname{sinkt}}{k}+O\left(1 / k^{\beta-\alpha+1}\right)
$$

and

$$
\int_{t}^{\pi} u^{\beta-\alpha} \cos k u d u=\frac{\pi \Gamma(\beta-\alpha+1)}{2 k^{\beta-\alpha+1}} \cos \frac{\pi}{2}(\beta-\alpha+1)-\frac{t^{\beta-\alpha} \operatorname{sinkt}}{k}+O\left(1 / k^{\beta-\alpha+1}\right) \text {, }
$$

where if $\alpha=B$ we may obviously omit the last term in both cases.

Proof. By integration by parts, we have

$$
\begin{aligned}
\int_{0}^{t} u^{\beta-\alpha} & \cos k u d u \\
& =\left[\frac{\sin k u}{k} u^{\beta-\alpha}\right]_{0}^{t}+\frac{\alpha+1-\beta}{k}\left[\int_{0}^{\pi / k} u^{\beta-\alpha} \frac{\sin k u}{u} d u+\int_{\pi / k}^{t} u^{\beta-\alpha-1} \sin k u d u\right) \\
& =\frac{t^{\beta-\alpha} \sin k t}{k}+\frac{\alpha+1-\beta}{k}\left\{\left(\frac{\pi}{k}\right)^{\beta-\alpha} \int_{\xi}^{\pi / k} \frac{\sin ^{3} u}{u} d u+\left(\frac{\pi}{k}\right)^{\beta-\alpha-1} \int_{\pi / k}^{n} \sin k u d u\right\} \\
& =\frac{t^{\beta-\alpha} \sin k t}{k}+o\left(1 / k^{\beta-\alpha+1}\right),
\end{aligned}
$$

where $0 \leq \xi \leq \pi / k \leq n \leq t$. The second formula is obvious by

$$
\frac{2}{\pi} \int_{0}^{\pi} u^{\beta-\alpha} \cos k u d u=\frac{\Gamma(\beta-\alpha+1)}{k^{\beta-\alpha+1}} \cos \frac{\pi}{2}(\beta-\alpha+1) .
$$

Proof of Theorem 1. We suppose $0<\alpha<1$, because we can treat $\alpha=0$ or 1 more easily (see [3], [10]). Since

$$
\begin{aligned}
A_{k}(x) & =\frac{2}{\pi} \int_{0}^{\pi} \varphi(t) \cos k t d t \\
& =\frac{2}{\pi \Gamma(1-\alpha)} \int_{0}^{\pi} \cos k t \int_{0}^{t}(t-u)^{-\alpha} d \Phi_{\alpha}(u) d t \\
& =\int_{0}^{\pi} \frac{2}{\pi \Gamma(1-\alpha)} \int_{u}^{\pi}(t-u)^{-\alpha} \cos k t d t d \Phi_{\alpha}(u),
\end{aligned}
$$

we have 
Summability factors

167

$$
\begin{aligned}
& \sum_{k=j}^{l}\left(\frac{P_{n-k}}{P_{n}}-\frac{P_{n-k-1}}{P_{n-1}}\right) \lambda_{k} \mu_{k} k^{\beta-\alpha} A_{k}(x) \quad(1 \leq j \leq \imath \leq n) \\
& =\int_{0}^{\pi} \cdot d \Phi \Phi^{(u)} \frac{2}{\pi \Gamma(1-\alpha)} \int_{u}^{\pi}(t-u)^{-\alpha}\left\{\sum_{k=j}^{l} P(n, k) \lambda_{k} \mu_{k} k^{\beta-\alpha} \cos k t\right\} d t \\
& =\int_{0}^{\pi} d \Phi(u) \frac{2}{\pi \Gamma(1-\alpha)} \int_{u}^{\pi}(t-u)^{-\alpha_{G-\alpha}}(n ; j, \tau, t) d t \\
& =\int_{0}^{\pi} H_{\beta-\alpha}(n ; j, \tau, u) d \Phi_{\alpha}(u)
\end{aligned}
$$

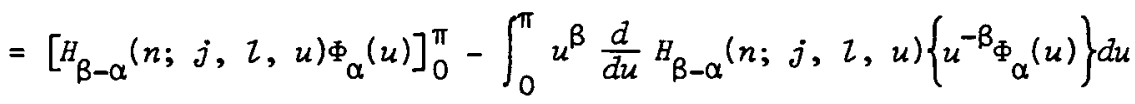

$$
\begin{aligned}
& =-\left[u^{-\beta_{\Phi}}(u) \int_{0}^{u} v^{\beta} \frac{d}{d v} H_{\beta-\alpha}(n ; j, \tau, v) d v\right]_{0}^{\pi} \\
& +\int_{0}^{\pi} \int_{0}^{u} v^{\beta} \frac{d}{d v} H_{\beta-\alpha}(n ; j, \tau, v) d v d\left\{u^{-\beta_{\Phi}}(u)\right\} \\
& =-\pi^{-\beta_{\Phi}}(\pi) I(n ; j, \tau, \pi)+\int_{0}^{\pi} I(n ; j, \imath, u) d\left\{u^{-\beta_{\Phi_{\alpha}}}(u)\right\} .
\end{aligned}
$$

If, in particular, we suppose that $\varphi(t)=t^{\beta-\alpha}$, in which case

$$
\Phi_{\alpha}(t)=\frac{1}{\Gamma(\alpha)} \int_{0}^{t}(t-u)^{\alpha-1} u^{\beta-\alpha} d u=\frac{\Gamma(\beta-\alpha+1)}{\Gamma(\beta+1)} t^{\beta}
$$

and

$$
A_{k}(x)=\frac{\Gamma(\beta-\alpha+1)}{k^{\beta-\alpha+1}} \cos \frac{\pi}{2}(\beta-\alpha+1),
$$

we get $d\left\{t^{-B_{\Phi}}(t)\right\}=0$ and

$$
I(n ; j, 2, \pi)=A \sum_{k=j}^{l} P(n, k) \frac{\lambda_{k} \mu_{k}}{k} \cos \frac{\pi}{2}(\beta-\alpha+1) .
$$

If $t_{n}$ denotes the $n$th $\left(N, p_{n}\right)$ mean of $\sum_{n=1}^{\infty} \lambda_{n} \mu_{n} n^{\beta-\alpha} A_{n}(x)$, then by (1.1) and the above calculations

https://doi.org/10.1017/S0004972700008650 Published online by Cambridge University Press 


$$
\begin{aligned}
t_{n}-t_{n-1} & =\sum_{k=1}^{n}\left\{\frac{P-k}{P_{n}}-\frac{P_{n-k-1}}{P_{n-1}}\right\} \lambda_{k} \mu_{k} k^{\beta-\alpha_{A}(x)} \\
& =A \sum_{k=1}^{n} P(n, k) \frac{\lambda_{k} \mu_{k}}{k} \cos \frac{\pi}{2}(\beta-\alpha+1)+\int_{0}^{\pi} I(n ; 2, n, u) d\left\{u^{\left.-\beta_{\Phi}(u)\right\} .}\right.
\end{aligned}
$$

Since, by (1.5) and Lemme 3,

$$
\begin{aligned}
\sum_{n=1}^{\infty}\left|\sum_{k=1}^{n} P(n, k) \frac{\lambda_{k} \mu_{k}}{k} \cos \frac{\pi}{2}(\beta-\alpha+1)\right| & \\
& \leq \sum_{k=1}^{\infty} \frac{\lambda_{k} \mu_{k}}{k}\left|\cos \frac{\pi}{2}(\beta-\alpha+1)\right| \sum_{n=k}^{\infty} P(n, k)<A,
\end{aligned}
$$

to prove our theorem it is enough to show that

$$
\sum_{n=1}^{\infty}\left|t_{n}-t_{n-1}\right|<A+\int_{0}^{\pi} \sum_{n=1}^{\infty}|I(n ; 2, n, u)|\left|d\left\{u^{-B_{\Phi_{\alpha}}(u)}\right\}\right|=O(1) .
$$

Thus it suffices for our purpose to prove that, uniformly in $0<u \leq \pi$,

$$
J=\sum_{n=1}^{\infty}|I(n ; \tau, n, u)|=O(\lambda(2 \pi / u)) \text {. }
$$

We divide $J$ into the following three parts:

(2.2) $J=\sum_{n=1}^{2 \tau+1}|I(n ; \tau, n, u)|+\sum_{n=2 \tau+2}^{\infty}|I(n ; \tau, \tau, u)|$

$$
=J_{1}+J_{2}+J_{3}, \quad+\sum_{n=2 \tau+2}^{\infty}|I(n, \tau+1, n, u)|
$$

say.

Using the first and second mean value theorems, we have 
(2.3) $h(u)=\left(\int_{u}^{u+\pi / k}+\int_{u+\pi / k}^{\pi} \cdot\right)(t-u)^{-\alpha} \cos k t d t$

$$
\begin{aligned}
& =\cos k \xi \int_{u}^{u+\pi / k}(t-u)^{-\alpha} d t+(k / \pi)^{\alpha} \int_{u+\pi / k}^{n} \cos k t d t \\
& (u \leq \xi \leq u+\pi / k \leq n \leq \pi) \\
& =\frac{k^{\alpha-1}}{(1-\alpha) \pi^{\alpha-1}} \cos k \xi+\frac{k^{\alpha-1}}{\pi^{\alpha}}[\sin k t]_{u+\pi / k}^{n} .
\end{aligned}
$$

Thus

(2.4) $H_{B-\alpha}(n ; j, \imath, u)$

$$
=\frac{2}{\pi^{1+\alpha} \Gamma(1-\alpha)} \sum_{k=j}^{2} P(n, k) \lambda_{k} \mu_{k} k^{\beta-1}\left\{\frac{\pi \cos k \xi}{1-\alpha}+\sin k n+\sin k u\right\} .
$$

Moreover, for some $\zeta \quad(0 \leq \zeta \leq u)$,

$$
\begin{aligned}
I(n ; j, \tau, u) & =\int_{0}^{u} v^{\beta} \frac{d}{d v} H_{B-\alpha}(n ; j, \tau, v) d v \\
& =u^{\beta} \int_{\zeta}^{u} \frac{d}{d v} H_{\beta-\alpha}(n ; j, \tau, v) d v \\
& =u^{\beta}\left[H_{\beta-\alpha}(n ; j, \tau, v)\right]_{\zeta}^{u} .
\end{aligned}
$$

Hence we get

$$
|I(n ; j, \imath, u)| \leq A u^{\beta} \sum_{k=j}^{Z} P(n, k) \lambda_{k} \mu_{k} k^{\beta-1} .
$$

Using (2.5),

$$
\begin{aligned}
J_{1} & \leq A u^{\beta} \sum_{n=1}^{2 \tau+1} \sum_{k=1}^{n} P(n, k) \lambda_{k} \mu_{k} k^{\beta-1} \\
& =A u^{\beta} \sum_{k=1}^{2 \tau+1} \cdot \lambda_{k} \mu_{k} k^{\beta-1} \sum_{n=k}^{2 \tau+1} P(n, k) \\
& \leq A u^{\beta} \lambda_{2 \tau+1} \sum_{k=1}^{2 \tau+1} k^{\beta-1}=O(\lambda(2 \pi / u)),
\end{aligned}
$$

by our assumptions and Lemma 3. For the same reasons, 


$$
\begin{aligned}
J_{2} & \leq A u^{\beta} \sum_{n=2 \tau+2}^{\infty} \sum_{k=1}^{\tau} P(n, k) \lambda_{k} \mu_{k} k^{\beta-1} \\
& =A u^{\beta} \sum_{k=1}^{\tau} \lambda_{k} \mu_{k} k^{\beta-1} \sum_{n=2 \tau+2}^{\infty} P(n, k) \\
& \leq A u^{\beta} \lambda_{\tau} \tau^{\beta}=O(\lambda(2 \pi / u)) .
\end{aligned}
$$

For the calculation of $J_{3}$, we see

$$
\begin{aligned}
& I(n ; j, \imath, u)=I(n ; j, \imath, \pi)-\int_{u}^{\pi} v^{\beta} \frac{d}{d v} H_{\beta-\alpha}(n ; j, \imath, v) d v \\
& =I(n ; j, \tau, \pi)-\left[v^{\beta} H_{\beta-\alpha}(n ; j, \tau, v)\right]_{u}^{\pi} \\
& +\beta \int_{u}^{\pi} v^{\beta-1} d v \frac{2}{\pi \Gamma(1-\alpha)} \int_{v}^{\pi}(t-v)^{-\alpha} G_{\beta-\alpha}(n ; j, \tau, t) d t \\
& =I(n ; j, \tau, \pi)+u^{\beta} H_{\beta-\alpha}(n ; j, \tau, u) \\
& +\frac{2 \beta}{\pi \Gamma(1-\alpha)} \int_{u}^{\pi} G_{\beta-\alpha}(n ; j, z, t) d t \int_{u}^{t} v^{\beta-1}(t-v)^{-\alpha} d v \\
& =I(n ; j, \tau, \pi)+u^{\beta} H_{B-\alpha}(n ; j, \tau, u) \\
& +\frac{2 \beta}{\pi \Gamma(1-\alpha)} \int_{u / \pi}^{1} w^{\beta-1}(1-w)^{-\alpha} d v \int_{\theta}^{\pi} t^{\beta-\alpha} G_{B-\alpha}(n ; j, \tau, t) d t \\
& \text { (by the second mean value theorem, where } u \leq \theta \leq \pi \text { ) } \\
& =A \sum_{k=j}^{l} P(n, k) \frac{\lambda_{k} \mu_{k}}{k} \cos \frac{\pi}{2}(\beta-\alpha+1)+u^{\beta} H_{\beta-\alpha}(n ; j, i, u) \\
& +o\left(\sum_{k=j}^{l} P(n, k) \lambda_{k} \mu_{k} k^{\beta-\alpha-1} \theta^{\beta-\alpha} \sin k \theta\right) \quad(\text { by Lemma } 4) .
\end{aligned}
$$

Thus, using (1.5) and (2.4), we have

$$
\text { (2.8) } \begin{aligned}
& J_{3} \leq A \sum_{n=2 \tau+2}^{\infty} u^{\beta}\left\{\left|G_{\beta-1}(n ; \tau+1, n, \xi)\right|+\left|\tilde{G}_{\beta-1}(n ; \tau+1, n, n)\right|\right. \\
& \left.+\left|\tilde{G}_{\beta-1}(n ; \tau+1, n, u)\right|\right\}+A \sum_{n=2 \tau+2}^{\infty} \theta^{\beta-\alpha}\left|\tilde{G}_{\beta-\alpha-1}(n ; \tau+1, n, \theta)\right|+A .
\end{aligned}
$$

Now we put 


$$
G_{\gamma}^{*}(n ; j, 2, \omega)=\sum_{k=j}^{l} P(n, k) \lambda_{k} \mu_{k} k^{\gamma} e^{i k \omega}
$$

where $\gamma=\beta-\varepsilon-1(\varepsilon=0$ or $\alpha)$ and $u \leq \omega \leq \pi$. Then, by (2.8), in order to prove that $J_{3}=O(\lambda(2 \pi / u))$, it is enough to prove that

$$
\text { (2.9) } \begin{aligned}
\omega^{\gamma+1} & \sum_{n=2 \tau+2}^{N}\left|G_{\gamma}^{*}(n, \tau+1, n, \omega)\right| \\
& \leq \omega^{\gamma+1}\left\{\sum_{n=2 \tau+2}^{N}\left|G_{\gamma}^{*}(n, \tau+1, m, \omega)\right|+\sum_{n=2 \tau+2}^{N}\left|G_{\gamma}^{*}(n ; m+1, n, \omega)\right|\right\} \\
& =K_{1}+K_{2}=O(\lambda(2 \pi / u)), \text { as } N \rightarrow \infty .
\end{aligned}
$$

Now using Lemma 1 , we get

$$
\begin{aligned}
\left|G^{*}(n ; j, \imath, \omega)\right| & \leq A \omega^{-1}\left[P(n, j) \lambda_{j} j_{j} j^{\gamma}+P(n, \imath) \lambda_{Z^{\mu} \eta^{2}{ }^{\gamma}}\right. \\
& \left.+\sum_{k=j}^{Z_{-1}}\left(\frac{P_{n-k-1}}{P_{n-1}}-\frac{P_{n-k}}{P_{n}}\right) \lambda_{k+1} \mu_{k+1}(k+1)^{\gamma}+\sum_{k=j}^{Z-1} P(n, k) \Delta\left(\frac{\lambda_{k} \mu_{k}}{k^{-\gamma}}\right)\right] .
\end{aligned}
$$

Hence

$K_{1}$

$\leq A \omega\left[\sum_{n=2 \tau+2}^{N} P(n, \tau+1) \lambda_{\tau+1} \mu_{\tau+1}(\tau+1)^{\gamma}+\sum_{n=2 \tau+2}^{N} \frac{{ }_{n} p_{n-m^{-}} P_{n}{ }^{P} n-m}{P_{n} P_{n-1}} \lambda_{m}{ }_{m} m^{\gamma}\right.$

$+\sum_{n=2 \tau+2}^{N} \sum_{k=\tau+1}^{m}\left(\frac{p_{n-k-1}}{P_{n-1}}-\frac{P_{n-k}}{P_{n}}\right) \lambda_{k+1} \mu_{k+1}(k+1)^{\gamma}$

$\left.+\sum_{n=2 \tau+2}^{N} \sum_{k=\tau+1}^{m} P(n, k) \Delta\left[\frac{\lambda_{k} \mu_{k}}{k-\gamma}\right)\right]$

$\leq A \omega^{\gamma}\left[\lambda_{\tau+1} \tau^{\gamma} \sum_{n=2 \tau+2}^{N} P(n, \tau+1)+\sum_{n=2 \tau+2}^{N} \frac{p_{n-m}}{P_{n-1}} \lambda_{m} \mu_{m} m^{\gamma}\right.$

$+\sum_{k=\tau+1}^{[N / 2]} \lambda_{k+1} \mu_{k+1}(k+1)^{\gamma} \sum_{n=2 k}^{N}\left(\frac{p_{n-k-1}}{P_{n-1}}-\frac{p_{n-k}}{P_{n}}\right)$

$\left.+\sum_{k=\tau+1}^{[N / 2]} \Delta\left(\frac{\lambda_{k} \mu_{k}}{k^{-\gamma}}\right) \sum_{n=2 k}^{N} P(n, k)\right] \leq$ 
172

Kôsi Kanno

$$
\begin{aligned}
& \leq A \lambda_{2 \tau}+A \omega \gamma\left[\sum_{n=2 \tau+2}^{N} \frac{P_{m}}{P_{m}} \lambda_{m} \mu_{m}{ }^{\gamma}+\sum_{k=\tau+1}^{N} \lambda_{k+1} \mu_{k+1}(k+1)^{\gamma} \frac{p_{k-1}}{P_{2 k-1}}+\sum_{k=\tau+1}^{N} \Delta\left(\frac{\lambda_{k} \mu_{k}}{k^{-\gamma}}\right)\right] \\
& \leq A \lambda_{2 \tau}+A \omega^{\gamma}\left[p_{\tau} \tau^{-\varepsilon} \sum_{n=\tau}^{\infty} \frac{n^{\gamma+\varepsilon} \lambda_{n} \mu_{n}}{P_{n}}+p_{\tau} \tau^{-\varepsilon} \sum_{k=\tau}^{\infty} \frac{k^{\gamma+\varepsilon} \lambda_{k} \mu_{k}}{P_{k}}+\lambda_{\tau+1}(\lambda+1)^{\gamma}\right] \\
& \text { because }\left\{p_{n}\right\} \text { and }\left\{\lambda_{k} \mu_{k} / k^{-\gamma}\right\} \text { are non-increasing) } \\
& \leq A \lambda_{2 \tau}+A \omega^{\gamma}\left(\tau^{\gamma+1} \lambda_{\tau} p_{\tau} / P_{\tau}+\tau^{\gamma} \lambda_{2 \tau}\right) \\
& =O(\lambda(2 \pi / u)) \text { (by }(1.4) \text { and } \tau p_{\tau} \leq P_{\tau} \text { ). } \\
& \text { Finally, by } P(n, k)=\left(P_{n} p_{n-k}-P_{n} P_{n-k}\right) / P_{n} P_{n-1} \text {, we get } \\
& K_{2} \leq A \omega^{\gamma+1} \sum_{n=2 \tau+2}^{N}\left|G_{\gamma}^{*}(n, m+1, n, \omega)\right| \\
& =A \omega^{\gamma+1} \sum_{n=2 \tau+2}^{N}\left|\sum_{k=m+1}^{n}\left(\frac{p_{n-k}}{P_{n-1}}-\frac{p_{n} P_{n-k}}{P_{n} P_{n-1}}\right) \lambda_{k} \mu_{k} k^{\gamma} e^{i k \omega}\right| \\
& \leq A \omega^{\gamma+1}\left[\sum_{n=2 \tau+2}^{N} \frac{1}{P_{n-1}}\left|\sum_{k=m+1}^{n} p_{n-k} \lambda_{k} \mu_{k} k^{\gamma} e^{i k \omega}\right|\right. \\
& \left.+\sum_{n=2 \tau+2}^{N} \frac{p_{n}}{P_{n}^{P} n-1}\left|\sum_{k=m+1}^{n} P_{n-k^{2}} \lambda_{k} \mu_{k} k^{\gamma} e^{i k \omega}\right|\right] \\
& =A \omega^{\gamma+1}\left[K_{21}+K_{22}\right] \text {, } \\
& \text { say. Since }\left\{\lambda_{k} \mu_{k} k^{\gamma}\right\} \text { is non-increasing, } \\
& K_{2 I} \leq \sum_{n=2 \nu}^{N} \frac{\lambda_{m} m_{m} m^{\gamma}}{P_{n-1}} \max _{m+1 \leq l \leq n}\left|\sum_{k=m+1}^{l} p_{n-k} e^{i k \omega}\right| \\
& \leq A P_{\nu} \nu^{-\varepsilon} \sum_{n=\nu}^{\infty} \frac{\lambda_{n}{ }_{n} n^{\gamma+\varepsilon}}{P_{n}} \leq A P_{\nu} \frac{\lambda_{\nu} \nu^{\gamma+1}}{P_{\nu}}=O\left(\nu^{\gamma+1} \lambda_{\tau}\right) \text {, }
\end{aligned}
$$

by Lemma 2 and Condition (1.4), where $v=[1 / \omega]$. Similarly,

$$
\begin{aligned}
K_{22} & \leq \sum_{n=2 v+2}^{N} \frac{p_{n}}{P_{n}^{P}{ }_{n-1}} \frac{P_{n-m}}{P_{n-m}} \lambda_{m}^{\mu} m^{m^{\gamma}} \max _{m+1 \leq l \leq n}\left|\sum_{k=m+1}^{l} p_{n-k} e^{i k \omega}\right| \\
& \leq A P_{v} \sum_{n=2 v+2}^{\infty} \frac{\lambda_{m}{ }_{m} m^{\gamma}}{P_{m}} \leq A P_{\nu} \cdot \lambda_{\nu} v^{\gamma+1} / P_{\nu}=o\left(v^{\gamma+1} \lambda_{\tau}\right) .
\end{aligned}
$$

https://doi.org/10.1017/S0004972700008650 Published online by Cambridge University Press 
For the case $P_{n} \leq A n^{\beta} \lambda_{n}$, the above estimations of $K_{1}, K_{2}$ also hold

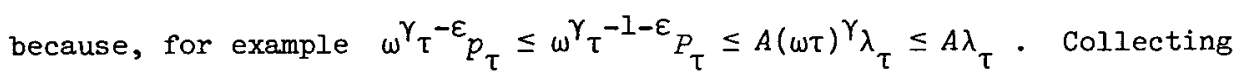
these estimations, we obtain (2.9).

Summing (2.2), (2.6), (2.7), and (2.9), we obtain (2.1).

This terminates the proof of Theorem 1.

Now we consider some applications of Theorem 1 .

COROLLARY 1. If $0<\alpha \leq \beta<1, \gamma \geq 0$, and

$$
\int_{0}^{\pi}\left\{\log \frac{2 \pi}{t}\right)^{\gamma}\left|d\left\{t^{-\beta_{\Phi}}(t)\right\}\right|<\infty \text {, }
$$

then the series $\sum_{n=1}^{\infty} n^{\beta-\alpha}(\log (n+1))^{\gamma-\delta_{A}}(t)$ is summable

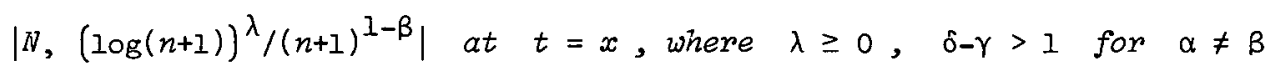
and $\lambda+\delta-\gamma>1$ for $\alpha=\beta$.

We can restate Theorem 1 in the following form.

COROLLARY 2. Suppose that $\left\{w_{n}\right\}$ is a positive sequence such that $P_{n} w_{n} / n$ is non-increasing, $P_{n} w_{n} / n^{\beta} \lambda_{n}$ is bounded and

$$
\begin{gathered}
\sum_{k=n}^{\infty} \frac{w_{k}}{k}=0\left(\frac{n^{\beta} \lambda_{n}}{P_{n}}\right), \\
\sum_{k=1}^{\infty} \frac{w_{k} P_{k}}{k^{1+\beta}}\left|\cos \frac{\pi}{2}(\beta-\alpha+1)\right|<\infty \quad(0 \leq \alpha \leq \beta \leq 1)
\end{gathered}
$$

hold. Then under the condition (1.6) we conclude that $\sum_{n=1}^{\infty} \frac{P_{n} w_{n}}{n^{\beta}} A_{n}(t)$ is sumabie $\left|N, p_{n}\right|$ at $t=x$. If $P_{n} \leq A n^{\beta} \lambda_{n}$, the right-hand side of (2.10) is replaced $O(1)$.

The next theorem is a special case of Corollary 2.

COROLLARY 3. Let $\left\{w_{n}\right\}$ be a positive sequence such that $w_{n} / n^{1-\alpha}$ is non-increasing and $\sum_{k=1}^{\infty} \frac{w_{k}}{k}$ is convergent. If $t^{-\alpha_{\Phi_{\alpha}}(t)}(0 \leq \alpha \leq 1)$ 
is a function of bounded variation in $(0, \pi)$, then the series $\sum_{n=1}^{\infty} w_{n} A_{n}(t)$ is sumnable $|C, \alpha|$ at $t=x$.

It is worth while to compare the results of Corollaries 2 and 3 with those due to Dikshit [2], Kishore [4], Lal [5], Mehrotra [8], Mohapatra, Das, and Srivastava [9], Prasad and Bhatt [12], and Vershney [13].

\section{Proofs and corollaries of Theorems 2 and 3}

We need the following lemmas, which are generalizations of Lemmas 3,4 , and 6 of Okuyama [11].

LEMMA 5. $\left\{\left(P_{n}-P_{n-k}\right) / k^{1-\alpha}\right\}(k=1,2, \ldots, n)$ is a non-decreasing sequence for $0 \leq \alpha \leq 1$.

Proof.

$$
\begin{aligned}
k^{1-\alpha}\left(P_{n}-P_{n-k-1}\right)- & (k+1)^{1-\alpha}\left(P_{n}-P_{n-k}\right) \\
& =\left\{k^{1-\alpha}-(k+1)^{1-\alpha}\right\} P_{n}-k^{1-\alpha_{P}}{ }_{n-k-1}+(k+1)^{1-\alpha}\left(P_{n-k}+P_{n-k-1}\right) \\
& \geq\left\{k^{1-\alpha}-(k+1)^{1-\alpha}\right\}\left(P_{n}-P_{n-k-1}\right)+(k+1)^{-\alpha}\left(P_{n}-P_{n-k-1}\right\} \\
& =\left(P_{n}-P_{n-k-1}\right)(k+1)^{-\alpha} k\left\{(i+1) / k^{\alpha}-1\right\} .
\end{aligned}
$$

Hence we have

$$
\frac{P_{n}-P}{n-k-1}{ }_{(k+1)^{1-\alpha}}-\frac{P_{n}{ }^{-P} n-k}{k^{1-\alpha}} \geq\left(P_{n}-P_{n-k-1}\right)\left\{(k+1)^{\alpha}-k^{\alpha}\right\} /(k+1) \geq 0 .
$$

LEMMA 6. Let $\left\{\Delta p_{n}\right\}$ be non-negative and non-increasing; then the sequence $\left\{\left(p_{n-k}-p_{n}\right) / k^{1-\alpha}\right\} \quad(k=1,2, \ldots, n)$ is non-decreasing for $0 \leq \alpha \leq 1$.

This follows similarly to Lemma 5.

LEMMA 7. Suppose that $t^{I-\alpha} / \lambda(t) \mu(t)$ and $t \lambda(t) \mu(t)$ are nondecreasing for $0 \leq \alpha \leq 1$. If $g(t)$ is a finction of bounded variation and 


$$
\int_{0}^{\pi} \frac{\lambda(C / t) \mu(C / t)}{t^{1+\alpha}}|g(t)| d t<\infty
$$

for some constant $C>\pi$, then the series

$$
\sum_{n=2}^{\infty} \frac{\lambda_{n} \mu_{n}\left|g\left(\theta_{n}\right)\right|}{n^{1 \cdot \cdot \alpha}}
$$

converges, where $\theta_{n}$ is a continuity point. of $g(t)$ such that $\pi /(n+1) \leq \theta_{n}<\pi / n, n \geq 2$.

The proof runs similarly to that of Lemma 6 of Okuyama [11].

Proof of Theorem 2. We may confine ourselves to the case $0<\alpha=\beta<1$. When $\alpha \neq \beta$, we only use $\Lambda(t)=t^{\beta-\alpha} \lambda(t)$ instead of $\lambda(t)$. Since

$$
\begin{aligned}
B_{k}(x) & =\frac{2}{\pi} \int_{0}^{\pi} \psi(t) \sin k t d t \\
& =\frac{2}{\pi}\left[\Psi_{1}(t) \sin k t\right]_{0}^{\pi}-\frac{2}{\pi} \int_{0}^{\pi} \Psi_{1}(t) k \cos k t d t \\
& =-\frac{2}{\pi} \int_{0}^{\pi} \cos k t d t \frac{k}{\Gamma(1-\alpha)} \int_{u}^{t}(t-u)^{-\alpha_{\Psi}}(u) d u \\
& =-\frac{2}{\pi \Gamma(1-\alpha)} \int_{0}^{\pi} \Psi_{\alpha}(u) d u \int_{0}^{\pi}(t-u)^{-\alpha_{k} \cos k t d t,}
\end{aligned}
$$

we get for the $n$th $\left(N, p_{n}\right)$ mean $\tilde{t}_{n}$ of $\sum_{n=1}^{\infty} \lambda_{n} \mu_{n}{ }_{n}(x)$,

$$
\begin{aligned}
& \tilde{t}_{n}-\tilde{t}_{n-1}=\sum_{k=1}^{n} P(n, k) \lambda_{k} \mu_{k} B_{k}(x) \\
& =-\int_{0}^{\pi} \Psi_{\alpha}(u) d u \frac{2}{\pi \Gamma(1-\alpha)} \int_{u}^{\pi}(t-u)^{-\alpha_{G_{1}}}(n ; \tau, n, t) d t \\
& =-\int_{0}^{\pi} H_{1}(n ; z, n, u) \Psi_{\alpha}(u) d u \text {. }
\end{aligned}
$$

Let $\theta_{n}$ be a continuity point of $\Psi_{\alpha}(u)$ such that $\pi /(n+1) \leq \theta_{n}<\pi / n$ for $n \geq 1$. Then 


$$
\begin{aligned}
\sum_{n=1}^{\infty}\left|\tilde{t}_{n}-\tilde{t}_{n-1}\right| & <A \sum_{n=1}^{\infty}\left\{\left|\int_{0}^{\theta} n\right|+\left|\int_{\theta_{n}}^{\pi}\right| H_{1}(n ; z, n, u) \Psi_{\alpha}(u) d u\right. \\
& =L_{1}+L_{2},
\end{aligned}
$$

say.

We define

$$
Q_{n}(u)= \begin{cases}H_{1}(n ; l, n, u) & \text { for } 0 \leq u<\theta_{n}, \\ 0 & \text { for } \theta_{n} \leq u \leq \pi .\end{cases}
$$

Since $Q_{n}(u)=0$ for $n \geq 2 \tau+1 \geq \pi / u$, we have by (2.3),

$$
\begin{aligned}
\sum_{n=1}^{\infty}\left|Q_{n}(u)\right| & \leq \sum_{n=1}^{2 \tau+1}\left|Q_{n}(u)\right| \\
& \leq A \sum_{n=1}^{2 \tau+1} \sum_{k=1}^{n} P(n, k) \lambda_{k} \mu_{k} k^{\alpha} \\
& \leq A \sum_{k=1}^{2 \tau+1} k \lambda_{k} \mu_{k} k^{\alpha-1} \sum_{n=k}^{2 \tau+1} P(n, k) \\
& \leq A \tau \lambda_{2 \tau+1} \mu_{2 \tau+1} \sum_{k=1}^{2 \tau+1} k^{\alpha-1} \\
& =O\left(\tau^{\alpha+1}{ }_{\lambda(2 \pi / u) \mu(2 \pi / u))}\right.
\end{aligned}
$$

by Lemma 3 and the non-decreasing property of $\left\{k \lambda_{k} \mu_{k}\right\}$. Thus, by (1.7), we have

$$
\begin{aligned}
L_{1} & \leq \int_{0}^{\pi}\left|\Psi_{\alpha}(u)\right| \sum_{n=1}^{\infty}\left|Q_{n}(u)\right| d u \\
& \leq A \int_{0}^{\pi} \frac{\lambda(2 \pi / u) \mu(2 \pi / u)}{u^{1+\alpha}}\left|\Psi_{\alpha}(u)\right| d u<\infty .
\end{aligned}
$$

Observing that 


$$
\begin{aligned}
\int_{\theta_{n}}^{u} h(v) d v & =\int_{\theta_{n}}^{u} d v \int_{\theta_{n}}^{\pi}(t-v)^{-\alpha} \cos k t d t-\int_{\theta_{n}}^{u} d v \int_{\theta_{n}}^{v}(t-v)^{-\alpha} \cos k t d t \\
& =\int_{\theta_{n}}^{\pi} \cos k t d t \int_{\theta_{n}}^{u}(t-v)^{-\alpha} d v-\int_{\theta_{n}}^{u} \cos k t d t \int_{t}^{u}(t-v)^{-\alpha} d v \\
& =-\frac{1}{1-\alpha} \int_{\theta_{n}}^{\pi}(t-u)^{1-\alpha} \cos k t d t+\frac{1}{1-\alpha} \int_{\theta_{n}}^{\pi}\left(t-\theta_{n}\right)^{1-\alpha} \cos k t d t \\
& +\frac{1}{1-\alpha} \int_{\theta_{n}}^{u}(t-u)^{1-\alpha} \cos k t d t,
\end{aligned}
$$

we get, by integration by parts and simple calculations

$$
\begin{array}{r}
-\frac{2 k}{\pi \Gamma(1-\alpha)} \int_{\theta_{n}}^{\pi} h(u) \Psi_{\alpha}(u) d u=\frac{2 k}{\pi(1-\alpha) \Gamma(1-\alpha)}\left\{-\int_{\theta_{n}}^{\pi} d \Psi(u) \int_{u}^{\pi}(t-u)^{1-\alpha} \cos k t d t\right. \\
\left.\quad-\Psi_{\alpha}\left(\theta_{n}\right) \int_{\theta_{n}}^{\pi}\left(t-\theta_{n}\right)^{1-\alpha} \cos k t d t\right\} \\
=\frac{2}{\pi \Gamma(1-\alpha)}\left\{\int_{\theta_{n}}^{\pi} d \Psi_{\alpha}(u) \int_{u}^{\pi}(t-u)^{-\alpha} \sin k t d t\right. \\
\left.\quad+\Psi_{\alpha}\left(\theta_{n}\right) \int_{\theta_{n}}^{\pi}\left(t-\theta_{n}\right)^{-\alpha} \sin k t d t\right\} .
\end{array}
$$

Hence

$$
\begin{aligned}
L_{2} & \leq \sum_{n=1}^{\infty}\left|\int_{\theta_{n}}^{\pi} \tilde{H}_{0}(n ; \tau, n, u) d \Psi_{\alpha}(u)\right|+\sum_{n=1}^{\infty}\left|\Psi_{\alpha}\left(\theta_{n}\right)\right|\left|\tilde{H}_{0}\left(n ; \tau, n, \theta_{n}\right)\right| \\
& =L_{21}+L_{22},
\end{aligned}
$$

say. Considering

(3.4) $\tilde{H}_{0}(n ; j, l, u)=\frac{2}{\pi^{1+\alpha} \Gamma(1-\alpha)} \sum_{k=j}^{l} P(n, k) \lambda_{k} \mu_{k} k^{\alpha-1}\left\{\frac{\pi}{1-\alpha} \sin k \xi\right.$

$$
-\cos k \eta-\cos k u\} \quad(\operatorname{see}(2.3))
$$

we have 


$$
\tilde{H}_{0}(n ; j, z, u)=0\left(\sum_{k=j}^{z} P(n, k) \lambda_{k} \mu_{k} k^{\alpha-1}\right) \text {. }
$$

Thus, using Lemmas 5,6 , and 7 , we get by the same calculations as those of $I_{21}$ of Okuyama [11],

$$
\begin{aligned}
L_{22} & \leq A \sum_{n=1}^{\infty}\left|\Psi_{\alpha}\left(\theta_{n}\right)\right| \sum_{k=1}^{n} P(n, k) \lambda_{k} \mu_{k} k^{\alpha-1} \\
& \leq A \sum_{n=2}^{\infty} \frac{\lambda_{n} \mu_{n}\left|\Psi_{\alpha}\left(\theta_{n}\right)\right|}{n^{1-\alpha}}<\infty .
\end{aligned}
$$

Next, we shall estimate $L_{21}$. We define

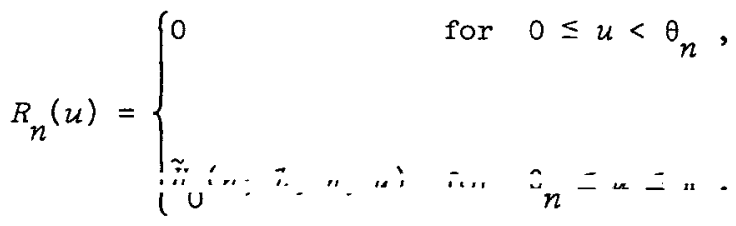

Since $R_{n}(u)=0$ for $n \leq \tau-1<\pi / u-1$, we have

$$
\begin{aligned}
L_{21} & \leq \sum_{n=1}^{\infty} \int_{0}^{\pi}\left|R_{n}(u)\right|\left|d \Psi_{\alpha}(u)\right| \\
& \leq \int_{0}^{\pi} \sum_{n=\tau}^{\infty}\left|R_{n}(u)\right|\left|d \Psi_{\alpha}(u)\right| .
\end{aligned}
$$

Hence, by (1.7), to prove the finiteness of $L_{2 l}$, it suffices to show that, uniformly in $0<t \leq \pi$,

$$
\begin{aligned}
M & =\sum_{n=\tau}^{\infty}\left|R_{n}(u)\right| \\
& =\sum_{n=\tau}^{\infty}\left|\tilde{H}_{0}(n ; \tau, n, u)\right|=O\left(u^{-\alpha} \lambda(2 \pi / u)\right) .
\end{aligned}
$$

We divide $M$ in the following form: 


$$
\begin{aligned}
M & \leq \sum_{n=\tau}^{2 \tau+1}\left|\tilde{H}_{0}(n ; \tau, n, u)\right|+\sum_{n=\frac{2 \tau+2}{\infty}\left|\tilde{H}_{0}(n ; \tau, \tau, u)\right|}+\sum_{n=2 \tau+2}^{\infty}\left|\tilde{H}_{0}(n ; \tau+1, n, u)\right| \\
& =M_{1}+M_{2}+M_{3},
\end{aligned}
$$

say. Then

$$
\begin{aligned}
M_{1} & \leq A \sum_{n=\tau}^{2 \tau+1} \sum_{k=1}^{n} P(n, k) \lambda_{k} \mu_{k} k^{\alpha-1} \\
& =A \sum_{k=1}^{\tau} \frac{\lambda_{k} \mu_{k}}{k^{1-\alpha}} \sum_{n=\tau}^{2 \tau+1} P(n, k)+A \sum_{k=\tau}^{2 \tau+1} \frac{\lambda_{k} \mu_{k}}{k^{1-\alpha}} \sum_{n=k}^{2 \tau+1} P(n, k) \\
& \leq A \lambda_{\tau} \sum_{k=1}^{\tau} k^{\alpha-1}+A \lambda_{2 \tau+1} \sum_{k=\tau}^{2 \tau+1} k^{\alpha-1}=o\left(\tau^{\alpha} \lambda_{2 \tau}\right) .
\end{aligned}
$$

Using (3.4) instead of (2.4), we may treat $M_{2}$ and $M_{3}$ by easier methods than those used for $J_{2}$ and $J_{3}$ in $\$ 2$. Thus we have (3.6). Combining (3.1), (3.2), (3.3), (3.5), and (3.6), Theorem 2 is completely proved.

Since the calculations to prove Theorem 3 are similar to those for Theorem 1, we omit them.

Using Theorems 2 and 3, we obtain several corollaries which are parallel to those of $\$ 2$ or Okuyama's paper [11].

We shall show one of them.

COROLLARY 4. If $0<\alpha \leq B<1$,

$$
\int_{0}^{\pi} t^{-1-B}\left(\log \frac{2 \pi}{t}\right)^{\gamma}\left|\Psi_{\alpha}(t)\right| d t<\infty \quad\left(\text { or } \Psi_{\alpha}(+0)=0\right)
$$

and

$$
\int_{0}^{\pi} t^{-\beta}\left(\log \frac{2 \pi}{t}\right)^{\gamma+1}\left|d \Psi_{\alpha}(t)\right|<\infty
$$

then the series $\sum_{n=2}^{\infty} n^{\beta-\alpha}(\log n)^{\gamma}{ }_{n}(t)$ is summable $\left|N,(\log n)^{\lambda} / n^{1-\beta}\right|$ at $t=x$, where $0<\gamma+1<\lambda$. 


\section{References}

[1] Shri Nivas Bhatt and Nand Kishore, "Absolute Nörlund summability of a Fourier series", Indian J. Math. 9 (1967), 259-267 (1968).

[2] G.D. Dikshit, "Absolute Nörlund summability factors for Fourier series", Pacific J. Math. 63 (1976), 371-379.

[3] Kôsi Kanno and Satoshi Watanabe, "On the absolute summability of factored Fourier series", Bulz. Yamagata Univ. Natur. Sci. 9 (1977), 197-203.

[4] Nand Kishore, "Absolute Nörlund summability of a factored Fourier series", Indian J. Math. 9 (1967), 123-136.

[5] Shiva Narain Lal, "On the absolute Nörlund summability factors of a Fourier series", Indian J. Math. 16 (1974), 1-22.

[6] Shiva Narain Lal, "On the absolute Nörlund summability of a series associated with a Fourier series", Publ. Inst. Math. (Beograd) 20 (34) $1976,169-178$.

[1] Kishi Matsumoto, "On absolute Cesàro summability of a series related to a Fourier series", Tôhoku Math. J. (2) 8 (1956), 205-222.

[8] Narain Das Mehrotra, "On the absolute Nörlund summability of a factored Fourier series", Proc. Japan. Acad. 41 (1965), 46-51.

[9] R.N. Mohapatra, G. Das and V.P. Srivastava, "On absolute summability factors of infinite series and their application to Fourier series", Proc. Cambridge Philos. Soc. 63 (1967), 107-118.

[10] Yasuo Okuyama, "On the absolute Nörlund summability factors of Fourier series", Bulz. Austral. Math. Soc. 12 (1975), 9-21.

[11] Yasuo Okuyama, "On the absolute Nörlund sumability factors of the conjugate series of a Fourier series", Tôhoku Math. J. (2) 28 (1976), 563-581.

[12] B.N. Prasad and S.N. Bhatt, "The summability factors of a Fourier series", Duke Hath. J. 24 (1957), 103-117. 
[13] O.P. Varshney, "On the absolute harmonic summability of a series related to a Fourier series", Proc. Amer. Math. Soc. 10 (1959), 784-789.

\author{
Mathematical Institute, \\ Yamagata University, \\ Yamagata, \\ Japan.
}

